\title{
Article \\ Omega-3 Fatty Acids Upregulate SIRT1/3, Activate PGC-1 $\alpha$ via Deacetylation, and Induce Nrf1 Production in 5/6 Nephrectomy Rat Model
}

\author{
Sung Hyun Son ${ }^{1,+}+\mathbb{D}$, Su Mi Lee ${ }^{2,+}\left(\mathbb{D}\right.$, Mi Hwa Lee ${ }^{3}$, Young Ki Son ${ }^{2}$, Seong Eun Kim ${ }^{2}$ and Won Suk An ${ }^{2, *(\mathbb{D}}$ \\ 1 Department of Internal Medicine, BHS Han Seo Hospital, Busan 48253, Korea; sohnseung@hanmail.net \\ 2 Department of Internal Medicine, Dong-A University, Busan 49201, Korea; sumilee@dau.ac.kr (S.M.L.); \\ kidney@dau.ac.kr (Y.K.S.); sekim@dau.ac.kr (S.E.K.) \\ 3 Department of Anatomy and Cell Biology, Dong-A University, Busan 49201, Korea; hero15p@nate.com \\ * Correspondence: anws@dau.ac.kr; Tel.: +82-51-240-2811 \\ + These authors contributed equally to this work.
}

Citation: Son, S.H.; Lee, S.M.; Lee, M.H.; Son, Y.K.; Kim, S.E.; An, W.S. Omega-3 Fatty Acids Upregulate SIRT1/3, Activate PGC- $1 \alpha$ via Deacetylation, and Induce Nrf1 Production in 5/6 Nephrectomy Rat Model. Mar. Drugs 2021, 19, 182. https://doi.org/10.3390/md19040182

Academic Editor:

Constantina Nasopoulou

Received: 19 March 2021

Accepted: 23 March 2021

Published: 26 March 2021

Publisher's Note: MDPI stays neutral with regard to jurisdictional claims in published maps and institutional affiliations.

Copyright: (c) 2021 by the authors. Licensee MDPI, Basel, Switzerland. This article is an open access article distributed under the terms and conditions of the Creative Commons Attribution (CC BY) license (https:// creativecommons.org/licenses/by/ $4.0 /)$.

\begin{abstract}
Mitochondrial dysfunction contributes to the pathogenesis of kidney injury related with cardiovascular disease. Peroxisome proliferator-activated receptor gamma coactivator-1 alpha (PGC$1 \alpha$ ) protects renal tubular cells by upregulating nuclear factor erythroid 2-related factor 2 (Nrf2). AMPactivated protein kinase (pAMPK)-mediated phosphorylation and sirtuin 1/3 (SIRT1/3)-mediated deacetylation are required for PGC- $1 \alpha$ activation. In the present study, we aimed to investigate whether omega-3 fatty acids (FAs) regulate the expression of mediators of mitochondrial biogenesis in 5/6 nephrectomy ( $\mathrm{Nx}$ ) rats. Male Sprague-Dawley rats were assigned to the following groups: sham control, Nx, and Nx treated with omega-3 FA. The expression of PGC-1 $\alpha$, phosphorylated PGC$1 \alpha$ (pPGC- $1 \alpha$ ), acetylated PGC- $1 \alpha$, and factors related to mitochondrial biogenesis was examined through Western blot analysis. Compared to the control group, the expression of PGC- $1 \alpha$, pAMPK, SIRT1/3, Nrf1, mTOR, and Nrf2 was significantly downregulated, and that of Keap 1, acetylated PGC- $1 \alpha$, and FoxO1/3, was significantly upregulated in the Nx group. These changes in protein expression were rescued in the omega-3 FA group. However, the expression of pPGC-1 $\alpha$ was similar among the three groups. Omega-3 FAs may involve mitochondrial biogenesis by upregulating Nrf1 and Nrf2. This protective mechanism might be attributed to the increased expression and deacetylation of PGC- $1 \alpha$, which was triggered by SIRT1/3.
\end{abstract}

Keywords: peroxisome proliferator-activated receptor gamma coactivator-1 alpha; nuclear respiratory factor 1; nuclear factor erythroid 2-related factor 2; sirtuin 1; sirtuin 3; omega-3 fatty acid

\section{Introduction}

Kidneys are involved in regulating several functions, including various metabolic pathways, water and electrolyte balance, blood pressure control, and production of several hormones. As these functions consume enormous energy, kidneys are rich in mitochondria [1]. Mitochondria can adapt to different metabolic conditions via regulating mechanistic target of rapamycin (mTOR) and AMP-activated protein kinase (AMPK)-mediated nutrient sensing pathways to maintain healthy mitochondrial population [2].

Among the studies on mitochondria, to date, there are only a few studies on mitochondrial biogenesis. Most studies related to mitochondrial biogenesis are based on non-renal tissue [3,4], and studies on kidneys are mainly related to acute kidney injury (AKI) animal models, proximal tubular cells, and diabetic nephropathy [5-7]. Although mitochondrial biogenesis may link to pathogenesis of mitochondrial dysfunction in non-diabetic chronic kidney disease (CKD), research on this topic is also very limited. CKD and mitochondrial biogenesis are related to cardiovascular disease [8,9]. Improving mitochondrial biogenesis may be beneficial for cardioprotection in CKD. 
A study indicating that omega-3 fatty acids (FAs) facilitate the biogenesis of mitochondria was conducted using non-kidney tissues $[3,10]$. A recent study demonstrated a promising role of omega-3 FAs in mitochondrial biogenesis in animal models with cardiovascular and neurodegenerative diseases [11]. Therefore, in the present study, we aimed to investigate mitochondrial biogenesis in kidneys of $5 / 6$ nephrectomy $(\mathrm{Nx})$ rats. Additionally, we evaluated the effect of omega-3 FAs on mitochondrial biogenesis using this model.

\section{Results}

\subsection{Changes in Kidney Function and Histological Findings}

Compared to the control group, blood urea nitrogen (BUN) level in the Nx and $\mathrm{Nx}$ treated with omega-3 FA groups were significantly increased (control group $17.7 \pm 1.5, \mathrm{Nx}$ group $77.7 \pm 28.4$, and Nx with omega-3 FA group $63.9 \pm 17.0 \mathrm{mg} / \mathrm{dL}, p=0.007$ ). Although there was no significant difference between the $\mathrm{Nx}$ and $\mathrm{Nx}$ treated with omega-3 FA groups, BUN levels were numerically lower in the Nx treated with omega-3 FA group. The serum creatinine (sCr) level among the three groups was similar to that of BUN (control group, $0.4 \pm 0.0 ;$ Nx group, $1.3 \pm 0.6$; omega-3 FA, $1.0 \pm 0.3 \mathrm{mg} / \mathrm{dL} ; p=0.008$ ). Compared to the control group, severe tubular dilatation and atrophy were observed using PAS staining in the Nx group (Supplementary Figure S1). Furthermore, the results showed that the Nx treated with omega-3 FA group exhibited less tubulointerstitial changes than the Nx group. The renal function and histological changes in the three groups have already been reported in a previous study [12].

\subsection{Changes in Factors Related to Mitochondrial Biogenesis}

\subsubsection{Nrf1 and Nrf2 Expression}

Compared to the control group, the Nx group showed significantly decreased expression level of nuclear respiratory factor 1 (Nrf1) and nuclear factor erythroid 2-related factor 2 (Nrf2). However, in the Nx treated with omega-3 FA group, the expression level of Nrf1 and Nrf2 was significantly increased compared to the Nx group, but did not reach the level of control group (Figure 1, Supplementary Figure S2). These results were also found in Nrf1 mRNA quantitative real-time PCR analysis (Supplementary Figure S3).

(A)
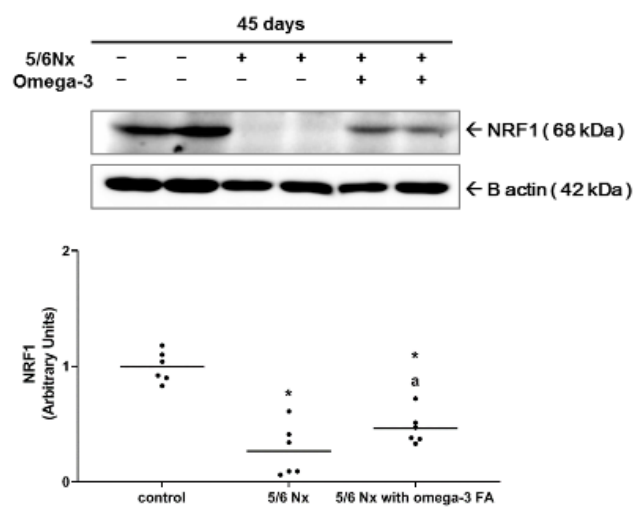

(B)
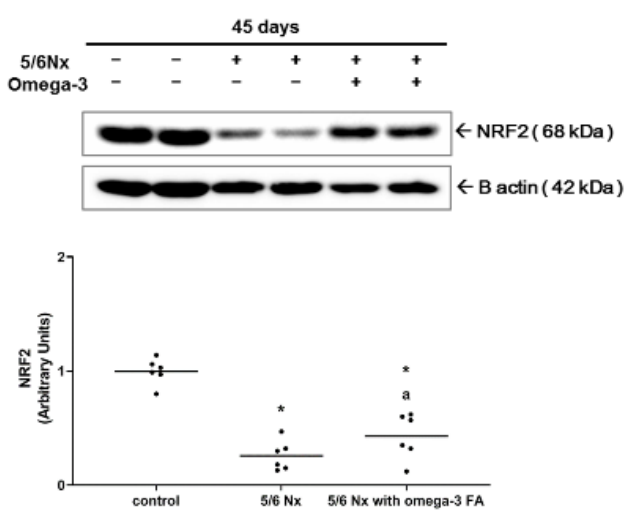

Figure 1. Expressions of (A) Nrf1 and (B) Nrf2 ( $n=6$ /group). Decreased Nrf 1 and Nrf2 expressions of $5 / 6$ nephrectomy group were improved by omega-3 FA supplementation. ${ }^{*} p$ value $<0.05$ (mean values are significantly different from control). ${ }^{a} p$ value $<0.05$ (mean values are significantly different from the Nx group).

\subsubsection{Changes in PGC- $1 \alpha$ Expression and Its Activity}

The expression of peroxisome proliferator-activated receptor gamma coactivator 1alpha (PGC- $1 \alpha$ ) in the Nx group was significantly decreased compared to the control group. The Nx treated with omega-3 FA group showed significantly increased PGC- $1 \alpha$ 
expression compared to the Nx group, but it did not reach the level of control group (Figure 2A, Supplementary Figure S4). These results were also found in PGC- $1 \alpha$ mRNA quantitative real-time PCR analysis (Supplementary Figure S5). To assess PGC-1 $\alpha$ activity, we evaluated the phosphorylation status (the ratio of phosphorylated PGC (pPGC)- $1 \alpha$ to PGC- $1 \alpha$ ) and acetylation status (the ratio of acetylated PGC- $1 \alpha$ to PGC- $1 \alpha$ ) of PGC- $1 \alpha$. The phosphorylation status of PGC- $1 \alpha$ in the Nx and Nx treated with omega-3 FA groups was not significantly different compared to the control group. However, the acetylation status of PGC- $1 \alpha$ was significantly increased only in the Nx group (Figure 2B). Moreover, the deacetylation status of PGC- $1 \alpha$ was significantly decreased only in the Nx group, but in the $\mathrm{Nx}$ treated with omega-3 FA group, it was not different from that in the control group.

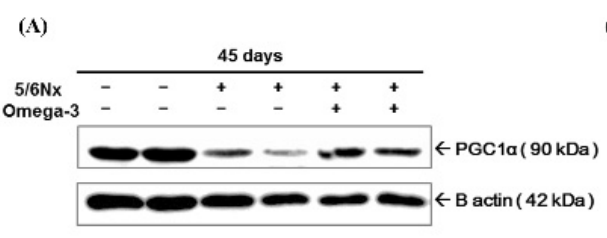

(B)
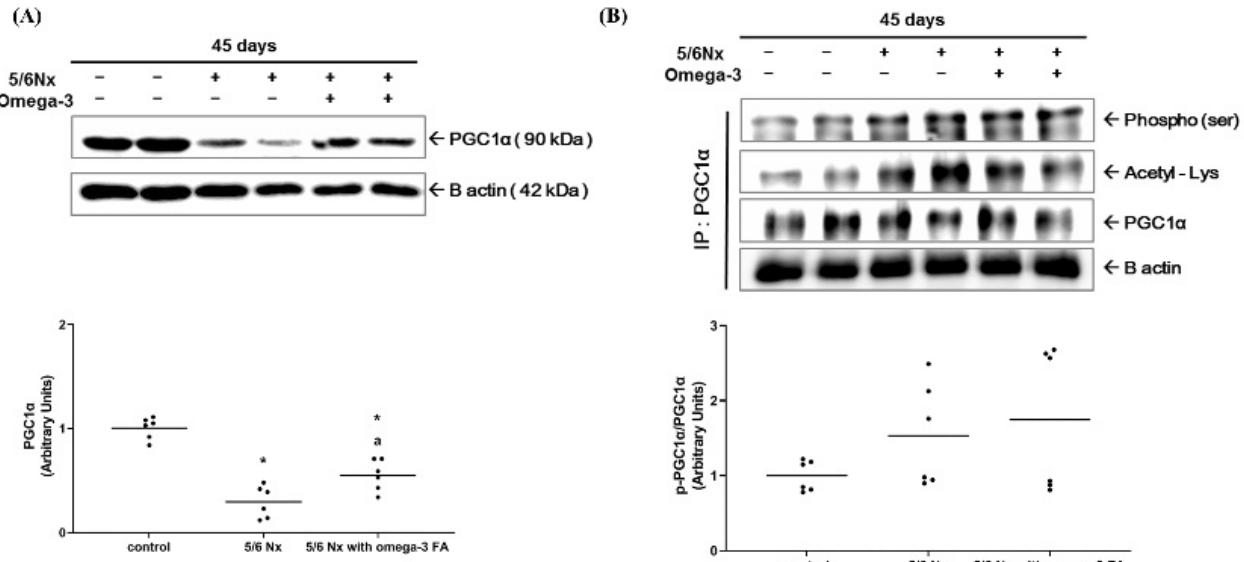
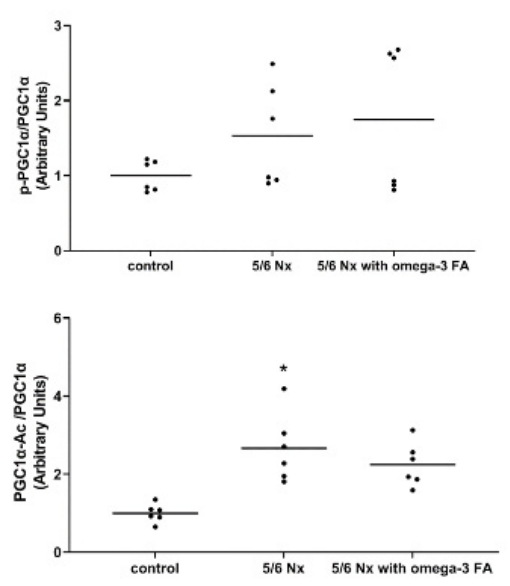

Figure 2. Changes in the (A) expression of PGC- $1 \alpha$ and (B) activity of PGC- $1 \alpha$ ( $n=6 /$ group). Decreased PGC- $1 \alpha$ expressions of $5 / 6$ nephrectomy group were improved by omega-3 FA supplementation. The deacetylation status of PGC- $1 \alpha$ was improved by omega-3 FA supplementation. p-PGC- $1 \alpha$, phosphorylated PGC- $1 \alpha$. PGC- $1 \alpha$-Ac, acetylated PGC- $1 \alpha .{ }^{*} p$ value $<0.05$ (mean values are significantly different from control). ${ }^{\mathrm{a}} p$ value $<0.05$ (mean values are significantly different from the Nx group).

\subsubsection{Expression of Factors Related to PGC- $1 \alpha$ Activity: pAMPK, SIRT1/3}

Compared to the control group, the Nx and Nx treated with omega-3 groups exhibited significantly lower levels of phosphorylated AMPK (the ratio of pAMPK to AMPK) (Figure 3A). Moreover, the Nx group exhibited significantly lower level of sirtuin 1 (SIRT1) expression compared to the control group (Figure 3B, Supplementary Figure S6). However, following the addition of omega-3 FA, SIRT1 expression was relatively increased, but this difference was not statistically significant. These results were found in SIRT1 mRNA quantitative real-time PCR analysis (Supplementary Figure S7). Conversely, sirtuin 3 (SIRT3) expression was significantly decreased in the Nx group compared to the control group, which was rescued significantly in the Nx treated with the omega-3 FA group (Figure 3C). 
(A)
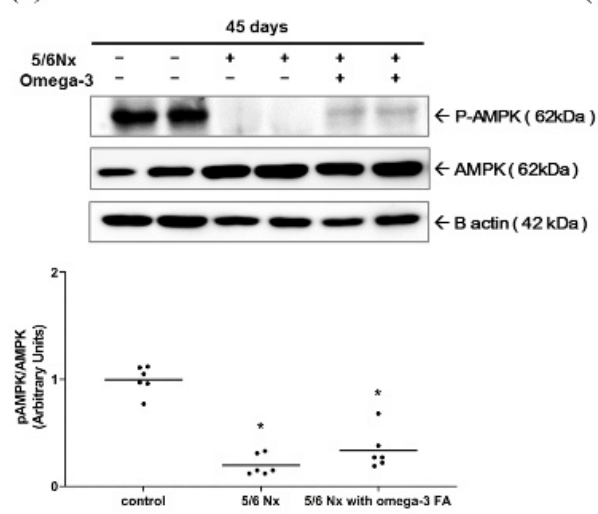

(B)

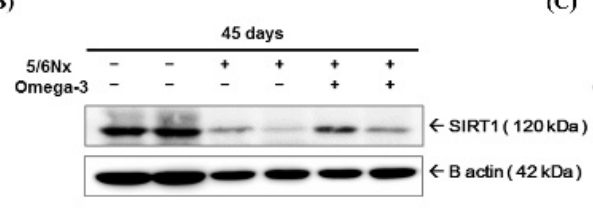

(C)

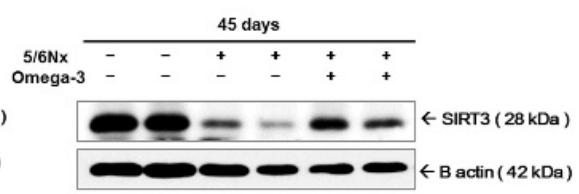

Figure 3. Changes in the expression of factors related to the function of PGC- $1 \alpha$ ( $n=6 /$ group): (A) phosphorylation of AMPK (the ratio of pAMPK to AMPK), (B) expression of SIRT1, and (C) SIRT3. Decreased SIRT3 expressions of $5 / 6$ nephrectomy group were improved by omega-3 FA supplementation. ${ }^{*} p$ value $<0.05$ (mean values are significantly different from control). ${ }^{a} p$ value $<0.05$ (mean values are significantly different from the Nx group).

\subsubsection{Keap1, mTOR, FoxO1, and FoxO3 Expression}

Compared to the control group, the expression of Kelch-like ECH-associated protein 1 (Keap1) and Forkhead box proteins $\mathrm{O} 1$ and $\mathrm{O} 3$ (FoxO1/3) was significantly increased in the Nx group. In the Nx treated with omega-3 FA group, the expression level of Keap1 and FoxO1/3 was significantly decreased compared to the Nx group (Figure 4C,E,F). Compared to the control group, the expression of mTOR was significantly decreased in the Nx group. However, in the Nx treated with omega-3 FA group, mTOR expression was rescued, but did not reach the level in the control group (Figure 4B).

(A)

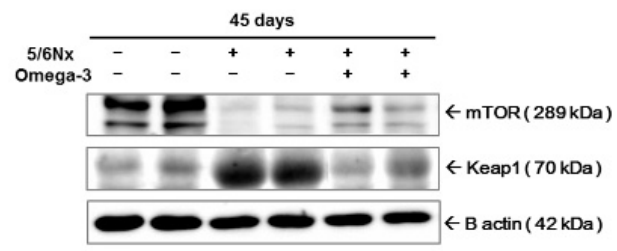

(D)

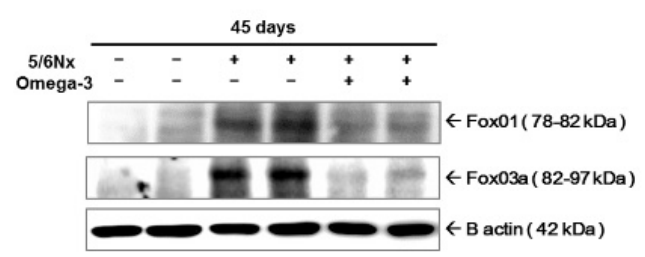

(B)

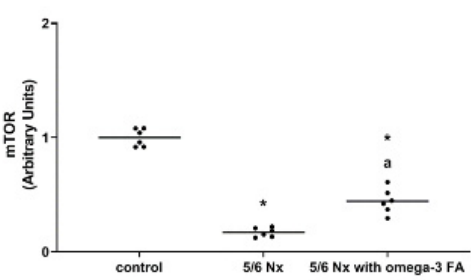

(E)

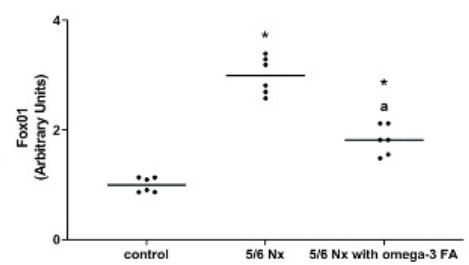

(C)

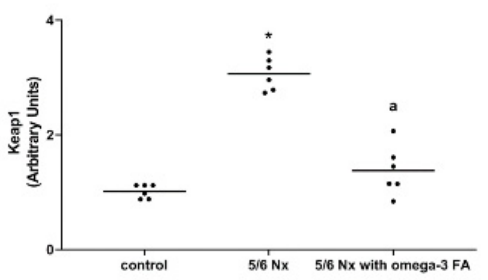

(F)

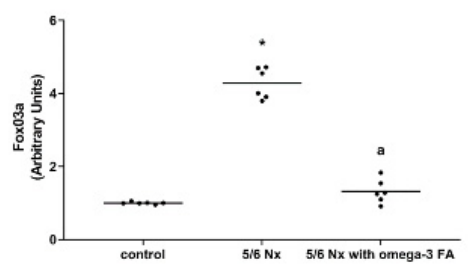

Figure 4. Changes in the expression of mTOR and Keap1 (A-C, $n=6 /$ group). Changes in the expression of FoxO1 and FoxO3 (D-F, $n=6$ /group). Decreased mTOR and increased Keap1, FoxO1, and FoxO3 expressions of 5/6 nephrectomy group were improved by omega-3 FA supplementation. ${ }^{*} p$ value $<0.05$ (mean values are significantly different from control). ${ }^{a} p$ value $<0.05$ (mean values are significantly different from the $\mathrm{Nx}$ group). 


\subsubsection{Content of Mitochondrial DNA (mtDNA)}

A significant reduction of mtDNA was observed in the Nx group compared to control group. However, the reduction amount of mtDNA was significantly recovered in the Nx-treated omega-3 Nx group (Supplementary Figure S9).

\section{Discussion}

PGC- $1 \alpha$, which is activated via phosphorylation and deacetylation, is the master regulator of mitochondrial biogenesis and energy production. We found a decrease in the expression and deacetylation of PGC- $1 \alpha$ in CKD model. It is noteworthy that decreased PGC- $1 \alpha$ deacetylation is the main mechanism contributing to dysfunctional mitochondrial biogenesis and reduced energy production in CKD. AMPK activates PGC- $1 \alpha$ via phosphorylating its threonine or serine residues [13,14]. AMPK is also activated upon phosphorylation when the AMP/ATP ratio is high. However, AMPK is known to be inactivated in CKD due to the impaired sensing of high AMP levels $[15,16]$. Similar with previous studies, we demonstrated that AMPK phosphorylation was significantly decreased in kidney tissue of the Nx group. Although pPGC-1/PGC-1 $\alpha$ appears to be increased to a certain extent in the Nx group, it is unlikely that the level of PGC- $1 \alpha$ phosphorylation is increased because of decreased PGC- $1 \alpha$ expression. Moreover, the Nx treated with omega-3 FA group also showed similar results as that of the Nx group, which indicates that omega-3 FA administration did not affect the phosphorylation of AMPK and PGC- $1 \alpha$. Deacetylation can occur in the entire sequence of PGC-1 $\alpha$, and SIRT1 and 3 play a major role in the process of deacetylation $[13,14]$. After omega-3 FA administration, the expression level of PGC-1 $\alpha$, SIRT1, and 3 increased, resulting in the rescue of deacetylated PGC- $1 \alpha$ to the same level as that of the normal control group. The rescue in the expression level of Nrf1 and Nrf2, which is demonstrated in the Nx treated with omega-3 FA group, is directly related to the increase in SIRT1 and 3 expression level, which further led to the deacetylation of PGC- $1 \alpha$.

Recent studies on mitochondria in kidney disease have shown that the increase in PGC$1 \alpha$ or SIRT expression level through genetic or pharmacological interventions improved kidney damage in AKI animal models $[5-7,17,18]$. The underlying mechanism involves the effect of improvement in FA oxidation or antioxidative response. A previous study reported that omega-3 FA administration improved mitochondrial FA beta-oxidation and exhibited antioxidant effects in a 5/6 Nx rat model [19]. There are also reports suggesting that omega-3 FA increases the expression of PGC-1 $\alpha$, Nrf1, and SIRT1 in muscle cells, macrophages, and nerve cells $[3,10,11]$. As the effects of omega-3 FAs on mitochondria in a CKD model have not been reported yet, we demonstrated that omega-3 FA is effective in recovering mitochondrial biogenesis-related molecules and mtDNA using a CKD model in this study.

Both mTOR and AMPK are involved in mitochondrial biogenesis, but their roles vary depending on the nutritional status. mTOR, a target of rapamycin complex 1 (mTOC1), can be activated via energy stimuli, such as amino acid or glucose, and can trigger pathways that lead to anabolic processes and mitochondrial biogenesis. Conversely, AMPK can be activated via hypoxia and nutritional deficit, which leads to the activation of catabolic process and mitochondrial biogenesis, and inhibits energy consumption through mTOC1 [2]. In this study, we not only demonstrated a decrease in pAMPK/AMPK, but also a decrease in mTOR in the CKD model. Furthermore, omega-3 FA administration led to a near recovery of mTOR expression but no recovery of the decrease in pAMPK/AMPK. Therefore, omega-3 FAs might alleviate decreased mitochondrial biogenesis by increasing mTOR expression in kidneys of a CKD animal model. However, further studies are required to be conducted to clarify whether mTOR is decreased in CKD patients, and how the increase in mTOR expression through omega-3 FA treatment will affect the mitochondrial health and CKD progression.

FoxO1 and 3 bind to PGC-1 $\alpha$ promoter and enhance its transcription [20,21]. However, in this study, FoxO1 and 3 were found to be increased in the Nx group and decreased in the control and Nx treated with omega-3 FA groups. Therefore, it is assumed that the 
expression of FoxO1 and 3 is relevant for the compensatory response to decrease PGC- $1 \alpha$ in CKD or increase PGC-1 $\alpha$ through omega-3 FA administration. Nrf2 plays an important role in cellular redox homeostasis and mitochondrial biogenesis. There are three ubiquitin ligase systems related to the Nrf2 degradation (Keap1, $\beta$-transducin repeats-containing protein, and E3 ubiquitin ligase synoviolin) [22]. In this study, we showed a significant decrease in Nrf2 and a significant increase in Keap1 in the Nx group compared to the control group.d However, in the Nx treated with omega-3 FA group, the expression of Keap1 was found to be decreased and Nrf2 was increased. The results suggest that the improved antioxidative effect of omega-3 FA administration in the CKD rat model may be related to the decrease in Keap1 expression and Nrf2 degradation, which might enhance mitochondrial biogenesis. Based on the results obtained in this study, further research is required to elucidate the causal relationship between FoxO1/3 and Nrf2 in the CKD environment, and the effect of omega-3 FA administration.

The molecular weight of Nrf2 was found at 68 kilodalton $(\mathrm{kDa})$ area in this study and other recent studies [23,24]. However, previous studies indicated biologically relevant molecular weight of Nrf2 is $95-110 \mathrm{kDa}$ with evidence $[25,26]$. We also found bands which were suspected as biologically relevant Nrf2 (Supplementary Figure S8). Further studies are necessary to find the molecular weight of biologically important Nrf2.

In previous studies, the changes in mitochondria on the 28th day after $5 / 6 \mathrm{Nx}$ have been reported, which indicated a decrease in gene or protein expression associated with beta oxidation, oxidative phosphorylation, and inner membrane structural protein, but there was no result related to PGC- 1 expression $[27,28]$. In this study, we confirmed that mitochondrial biogenesis-related molecules and mtDNA reflecting mitochondrial biogenesis were modulated during the chronic stage, as the changes were observed on the 45th day after 5/6 Nx in rats. Despite the histological and biological improvements, the omega-3 FA treatment did not result in functional recovery of kidneys compared to the $\mathrm{Nx}$ model. Therefore, further research is required to determine whether omega-3 FAs are effective in restoring kidney function by inducing effects that might improve mitochondrial function.

There are several limitations in this this study. First, we did not evaluate citrate synthase activity and superoxide formation in the mitochondrial respiration chain. Second, we did not experiment which FA components of Omacor are mainly affected on mitochondrial biogenesis-related molecules. Third, we did not demonstrate a clear mechanism for improving mitochondrial biogenesis-related molecules. We just suggest that anti-inflammatory effect, reducing oxidative stress and omega- 3 fatty acid itself as metabolic compounds, may be related with the mechanism.

\section{Materials and Methods Mitochondrial Biogenesis}

\subsection{Animals and Experimental Design}

Studies were conducted on male Sprague-Dawley rats (9-weeks-old) purchased from Japan SLC, Inc. (Shizuoka, Japan). All rats were subjected to either sham control or $5 / 6 \mathrm{Nx}$. All procedures involving animals were performed in accordance with approval of Dong-A University's Institutional Animal Care Committee (DIACUC-14-4). Rats were kept under standard condition and allowed free access to tap water and standard diet.

All rats were assigned to the following groups: group 1 (sham control, $n=6$ ), control rats kept on saline $(1 \mathrm{~mL} / \mathrm{kg}$ /day by gastric lavage); group 2 (Nx group, $n=6)$, $\mathrm{Nx}$ rats kept on saline ( $1 \mathrm{~mL} / \mathrm{kg} /$ day by gastric lavage); group 3 (omega-3 FA group), $\mathrm{Nx}$ rats treated with omega-3 FAs. The dose and administration route of omega-3 FAs (Omacor, $300 \mathrm{mg} / \mathrm{kg}$ /day by gastric gavage, Pronova Biocare, Sandefjord, Norway) were determined based on previous studies [19]. Omacor is marine-originated omega-3 FA and is made up of $460 \mathrm{mg}$ eicosapentaenoic acid (EPA) and $380 \mathrm{mg}$ of docosahexaenoic acid (DHA) in $1 \mathrm{~g}$ of Omacor. Omacor is usually prescribed for secondary prevention after myocardial infarction and hypertriglyceridemia treatment [29]. Two omega-3 FAs (EPA plus DHA) are major $(84 \%)$ and active components of Omacor, which are pharmaceutically prepared 
compositions overcoming minor components (16\%). We chose Omacor for omega-3 FA supplementation with anti-inflammatory and reducing oxidative stress, because it decreased the risk of cardiovascular disease in the clinical studies [30]. The rats were fed evenly and their weight was observed daily. Rats were allowed free access to tap water for 15 weeks after surgery, and then euthanized under diethyl ether anesthesia. At death, an aortic blood sample was collected and then centrifuged at $3000 \mathrm{rpm}$ for $10 \mathrm{~min}$. To identify changes in kidney function, $\mathrm{BUN}$ and $\mathrm{sCr}$ levels were measured using an automatic analyzer (Roche, Germany).

\subsection{Histopathologic Examination}

Formalin-fixed kidney tissues were embedded in paraffin, cut into slices $(4 \mu \mathrm{m})$, and stained with periodic acid-Schiff. Histopathologic changes were observed with Aperio ScanScope (Aperio Technologies, Vista, CA, USA).

\subsection{RNA Isolation and Quantitative Real-Time PCR Analysis}

Total RNA was extracted from kidney tissues using TRIzol reagent. Then, $1 \mu \mathrm{g}$ of total RNA was converted into single-stranded cDNA using M-MLV cDNA synthesis kit (Enzynomics, Daejeon, Korea). Primers were designed from respective gene sequences using Primer3 and mfold software. For quantitative real-time PCR analysis, cDNA was subjected to PCR amplification using gene-specific primers: rat PGC $1 \alpha 5^{\prime}$ - CCG AGA ATT CAT GGA GCA AT-3' (sense), 5'- GTG TGA GGA GGG TCA TCG TT-3' (antisense); rat Nrf1 5' - GTT TCA TGG ACC CAA GCA TT-3' (sense), 5' - GGT GGC CTG AGT TTG TGT CT-3' (antisense); rat SIRT3, 5' - GAG ACT TGG TGG GGT CCT TT -3' (sense), 5' - ATC CTG CAG CTC TTG TGT CC -3' (antisense); rat SIRT1, 5' - CAG GTT GCA GGA ATC CAA AG - $3^{\prime}$ (sense), $5^{\prime}$ - CTC CAC GAA CAG CTT CAC AA - $3^{\prime}$ (antisense); rat $\beta$-actin, $5^{\prime}$ GCG CAA GTA CTC TGT GTG GA -3' (sense), 5' - CAT CGT ACT CCT GCT TGC TG -3' (antisense). Real-time PCR was performed using SYBR Green PCR Master Mix (Applied Biosystems, Foster City, CA, USA) with an ABI 7500 instrument (Applied Biosystems, Waltham, MA, USA).

\subsection{Western Blotting and Immunoprecipitation}

Western blotting was analyzed as described previously with slight modifications [10]. Kidney tissues were homogenized by lysis buffer (PRO-PREP protein extraction solution), incubated $\left(30 \mathrm{~min}\right.$ at $\left.4^{\circ} \mathrm{C}\right)$, and centrifuged $\left(14,000 \mathrm{rpm}\right.$ for $20 \mathrm{~min}$ at $\left.4{ }^{\circ} \mathrm{C}\right)$. The concentration of protein was determined by Bradford protein assay reagent (Bio-Rad, Hercules, CA, USA). Equal protein sample was loaded on a 7.5-15\% SDS-PAGE and transferred to a nitrocellulose membrane (Amersham Pharmacia Biotech, Piscataway, NJ, USA). Then, proteins were immunoblotted with each antibody. Antibodies against PGC-1 $\alpha$ and SIRT1 were obtained from Santa Cruz Biotechnology (Santa Cruz, CA, USA). Antibodies against AMPK, pAMPK, Nrf1, SIRT3, FoxO1, FoxO3a, and mTOR were purchased from Cell Signaling Technology (Danvers, MA, USA). Antibodies against Nrf2, Keap1 (monomer), and $\beta$-actin were purchased from Abcam (Cambridge, MA, USA) and Sigma (St. Louis, MO, USA). For immunoprecipitation, a total of $500 \mu \mathrm{g}$ protein was incubated with PGC-1 $\alpha$ antibodies (Santa Cruz Biotechnology, Santa Cruz, CA, USA) and protein A/G plus-agarose beads (Santa Cruz Biotechnology, Santa Cruz, CA, USA), and allowed to mix at $4{ }^{\circ} \mathrm{C}$ overnight. The immunoprecipitates were then separated using SDS-PAG electrophoresis and immunoblotted using the acetyl-lysine (Cell Signaling Technology, Beverly, MA, USA) and phosphoserine (MerckMillipore, Burlington, MA, USA) antibodies. The membranes were subsequently incubated with horseradish peroxidase-conjugated secondary antibody for $60 \mathrm{~min}$ at room temperature. Protein levels were standardized by $\beta$-actin (ImageJ version $1.48 \mathrm{q}$ ). Western blotting and immunoprecipitation analysis with antibodies was performed using the Super Signal West Pico enhanced chemiluminescence substrate (Thermo Scientific, Hudson, NH, USA) and detected using LAS-3000 Plus (Fuji Photo Film, Tokyo, Japan). 


\subsection{Measurement of mtDNA Content}

The qPCR was used to determine relative content of mtDNA. Reaction was performed via SYBR Green chemistry using 3 ng of total DNA as a template and the following primers: rat mtDNA $5^{\prime}$-GGTTCTTACTTCAGGGCCATCA-3' (sense), 5' -TGATTAGACCCGTTACCA TCGA- $3^{\prime}$ (antisense); rat $\beta$-actin, $5^{\prime}$ - CCCAGCCATGTACGTAGCCA - $3^{\prime}$ (sense), $5^{\prime}$ - CGTCTCCGGAGTCCATCAC $-3^{\prime}$ (antisense). The mtDNA content relative to the nuclear DNA was reported in [31].

\subsection{Statistical Analysis}

Statistical analyses were performed using the SPSS 18.0 software (IBM Corp., Armonk, NY, USA). Results are expressed as mean \pm SD. The means for the groups were compared by analysis of variance followed by Tukey's multiple comparison. $p<0.05$ was considered statistically significant.

\section{Conclusions}

Conclusively, significant modulations in the expression of mediators related to mitochondrial biogenesis were observed in a CKD rat model. Omega-3 FA may improve mitochondrial biogenesis by upregulating Nrf1 and Nrf2. This protective mechanism may be initiated through an increase in PGC- $1 \alpha$ expression and deacetylation of PGC- $1 \alpha$, which was triggered by increased SIRT1/3 production (Figure 5).

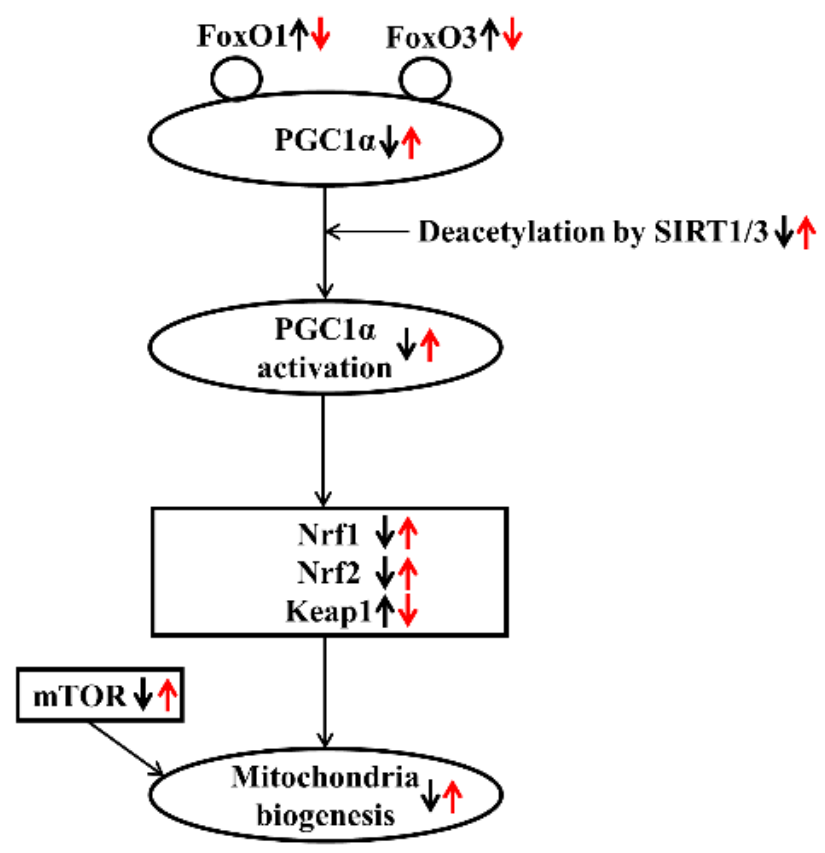

Figure 5. Effect of omega-3 FA on mitochondrial biogenesis in CKD. Bold black arrows indicate the expression of mediators related to mitochondrial biogenesis in a CKD rat model. Red arrows indicate the expression of mediators after omega-3 FA administration. Up/down arrows indicate an increase and decrease in the expression of mediators.

Supplementary Materials: The following are available online at https:/ /www.mdpi.com/1660-339 7/19/4/182/s1, Figure S1: Morphological changes in the kidney among three groups representing renal function. Figure S2: Expression of Nrf1 and Nrf2 on full length gels. Figure S3: Expression of Nrf1 by real time PCR among three groups. Figure S4: Expression of phosphorylated PGC- $1 \alpha$, acetylated PGC- $1 \alpha$, and PGC- $1 \alpha$ on full length gels. Figure S5: Expression of PGC- $1 \alpha$ by real time PCR among three groups. Figure S6: Expression of SIRT1 on full length gels. Figure S7: Expression of 
SIRT1 by real time PCR among three groups. Figure S8: Expression suspected as biologically relevant Nrf2 (96kDa) on full length gels. Figure S9: Relative content of mtDNA.

Author Contributions: W.S.A. designed the study. S.H.S., S.M.L., M.H.L. and W.S.A. conducted the study and analyzed the data. S.H.S., S.M.L., M.H.L., Y.K.S., S.E.K. and W.S.A. wrote the manuscript. All authors reviewed and approved the final version of the manuscript.

Funding: This study was supported by the Dong-A University research fund.

Institutional Review Board Statement: The study was conducted according to the guidelines of the Declaration of Helsinki, and approved by Dong-A University's Institutional Animal Care Committee (DIACUC-14-4).

Informed Consent Statement: Not applicable.

Data Availability Statement: The data presented in this study are available on request from the corresponding author.

Conflicts of Interest: The authors declare no conflict of interest.

\section{References}

1. Bhatia, D.; Capili, A.; Choi, M.E. Mitochondrial dysfunction in kidney injury, inflammation, and disease: Potential therapeutic approaches. Kidney Res. Clin. Pract. 2020, 39, 244-258. [CrossRef]

2. Bhargava, P.; Schnellmann, R.G. Mitochondrial energetics in the kidney. Nat. Rev. Nephrol. 2017, 13, 629-646. [CrossRef]

3. Lee, M.S.; Shin, Y.; Moon, S.; Kim, S.; Kim, Y. Effects of Eicosapentaenoic Acid and Docosahexaenoic Acid on Mitochondrial DNA Replication and PGC-1alpha Gene Expression in $\mathrm{C}_{2} \mathrm{C}_{12}$ Muscle Cells. Prev. Nutr. Food Sci. 2016, 21, 317-322. [CrossRef]

4. Park, J.S.; Davis, R.L.; Sue, C.M. Mitochondrial Dysfunction in Parkinson's Disease: New Mechanistic Insights and Therapeutic Perspectives. Curr. Neurol. Neurosci. Rep. 2018, 18, 21. [CrossRef]

5. Yuan, Y.; Huang, S.; Wang, W.; Wang, Y.; Zhang, P.; Zhu, C.; Ding, G.; Liu, B.; Yang, T.; Zhang, A. Activation of peroxisome proliferator-activated receptor-gamma coactivator 1alpha ameliorates mitochondrial dysfunction and protects podocytes from aldosterone-induced injury. Kidney Int. 2012, 82, 771-789. [CrossRef]

6. Jesinkey, S.R.; Funk, J.A.; Stallons, L.J.; Wills, L.P.; Megyesi, J.K.; Beeson, C.C.; Schnellmann, R.G. Formoterol restores mitochondrial and renal function after ischemia-reperfusion injury. J. Am. Soc. Nephrol. 2014, 25, 1157-1162. [CrossRef]

7. Garrett, S.M.; Whitaker, R.M.; Beeson, C.C.; Schnellmann, R.G. Agonism of the 5-hydroxytryptamine 1F receptor promotes mitochondrial biogenesis and recovery from acute kidney injury. J. Pharmacol. Exp. Ther. 2014, 350, 257-264. [CrossRef]

8. Siasos, G.; Tsigkou, V.; Kosmopoulos, M.; Theodosiadis, D.; Simantiris, S.; Tagkou, N.M.; Tsimpiktsioglou, A.; Stampouloglou, P.K.; Oikonomou, E.; Mourouzis, K.; et al. Mitochondria and cardiovascular diseases-from pathophysiology to treatment. Ann. Transl. Med. 2018, 6, 256. [CrossRef] [PubMed]

9. Bigelman, E.; Cohen, L.; Aharon-Hananel, G.; Levy, R.; Rozenbaum, Z.; Saada, A.; Keren, G.; Entin-Meer, M. Pathological presentation of cardiac mitochondria in a rat model for chronic kidney disease. PLoS ONE 2018, 13, e0198196. [CrossRef]

10. Xue, B.; Yang, Z.; Wang, X.; Shi, H. Omega-3 polyunsaturated fatty acids antagonize macrophage inflammation via activation of AMPK/SIRT1 pathway. PLoS ONE 2012, 7, e45990. [CrossRef]

11. De Oliveira, M.R.; Nabavi, S.M.; Jardim, F.R. Omega-3 polyunsaturated fatty acids and mitochondria, back to the future. Trends Food Sci. Technol. 2017, 67, 76-92. [CrossRef]

12. Lee, H.W.; Lee, S.M.; Lee, M.H.; Son, Y.K.; Kim, S.E.; An, W.S. Effect of Omega-3 Fatty Acid on STAMP2 Expression in the Heart and Kidney of 5/6 Nephrectomy Rat Model. Mar. Drugs 2018, 16, 398. [CrossRef]

13. Fernandez-Marcos, P.J.; Auwerx, J. Regulation of PGC-1alpha, a nodal regulator of mitochondrial biogenesis. Am. J. Clin. Nutr. 2011, 93, S884-S890. [CrossRef] [PubMed]

14. Ishimoto, Y.; Inagi, R. Mitochondria: A therapeutic target in acute kidney injury. Nephrol. Dial. Transplant. 2016, 31, 1062-1069. [CrossRef] [PubMed]

15. Jeon, S.M. Regulation and function of AMPK in physiology and diseases. Exp. Mol. Med. 2016, 48, e245. [CrossRef]

16. Kikuchi, H.; Sasaki, E.; Nomura, N.; Mori, T.; Minamishima, Y.A.; Yoshizaki, Y.; Takahashi, N.; Furusho, T.; Arai, Y.; Mandai, S.; et al. Failure to sense energy depletion may be a novel therapeutic target in chronic kidney disease. Kidney Int. 2019, 95, 123-137. [CrossRef]

17. Kang, H.M.; Ahn, S.H.; Choi, P.; Ko, Y.A.; Han, S.H.; Chinga, F.; Park, A.S.; Tao, J.; Sharma, K.; Pullman, J.; et al. Defective fatty acid oxidation in renal tubular epithelial cells has a key role in kidney fibrosis development. Nat. Med. 2015, 21, 37-46. [CrossRef]

18. Zhang, L.; Liu, J.; Zhou, F.; Wang, W.; Chen, N. PGC-1alpha ameliorates kidney fibrosis in mice with diabetic kidney disease through an antioxidative mechanism. Mol. Med. Rep. 2018, 17, 4490-4498. [CrossRef] [PubMed]

19. An, W.S.; Kim, H.J.; Cho, K.H.; Vaziri, N.D. Omega-3 fatty acid supplementation attenuates oxidative stress, inflammation, and tubulointerstitial fibrosis in the remnant kidney. Am. J. Physiol. Renal. Physiol. 2009, 297, F895-F903. [CrossRef] 
20. Borniquel, S.; Garcia-Quintans, N.; Valle, I.; Olmos, Y.; Wild, B.; Martinez-Granero, F.; Soria, E.; Lamas, S.; Monsalve, M. Inactivation of Foxo3a and subsequent downregulation of PGC-1 alpha mediate nitric oxide-induced endothelial cell migration. Mol. Cell. Biol. 2010, 30, 4035-4044. [CrossRef]

21. Daitoku, H.; Yamagata, K.; Matsuzaki, H.; Hatta, M.; Fukamizu, A. Regulation of PGC-1 promoter activity by protein kinase B and the forkhead transcription factor FKHR. Diabetes 2003, 52, 642-649. [CrossRef]

22. Holmstrom, K.M.; Kostov, R.V.; Dinkova-Kostova, A.T. The multifaceted role of Nrf2 in mitochondrial function. Curr. Opin. Toxicol. 2016, 1, 80-91. [CrossRef] [PubMed]

23. Hao, Y.; Miao, J.; Liu, W.; Peng, L.; Chen, Y.; Zhong, Q. Formononetin protects against cisplatininduced acute kidney injury through activation of the PPARalpha/Nrf2/HO1/NQO1 pathway. Int. J. Mol. Med. 2021, 47, 511-522. [CrossRef] [PubMed]

24. Arellano-Buendia, A.S.; Castaneda-Lara, L.G.; Loredo-Mendoza, M.L.; Garcia-Arroyo, F.E.; Rojas-Morales, P.; Arguello-Garcia, R.; Juarez-Rojas, J.G.; Tapia, E.; Pedraza-Chaverri, J.; Sanchez-Lozada, L.G.; et al. Effects of Allicin on Pathophysiological Mechanisms during the Progression of Nephropathy Associated to Diabetes. Antioxidants 2020, 9, 1134. [CrossRef] [PubMed]

25. Lau, A.; Tian, W.; Whitman, S.A.; Zhang, D.D. The predicted molecular weight of Nrf2: It is what it is not. Antioxid. Redox Signal. 2013, 18, 91-93. [CrossRef] [PubMed]

26. Taguchi, K.; Takaku, M.; Egner, P.A.; Morita, M.; Kaneko, T.; Mashimo, T.; Kensler, T.W.; Yamamoto, M. Generation of a New Model Rat: Nrf2 Knockout Rats Are Sensitive to Aflatoxin B1 Toxicity. Toxicol. Sci. 2016, 152, 40-52. [CrossRef]

27. Aparicio-Trejo, O.E.; Tapia, E.; Sanchez-Lozada, L.G.; Pedraza-Chaverri, J. Mitochondrial bioenergetics, redox state, dynamics and turnover alterations in renal mass reduction models of chronic kidney diseases and their possible implications in the progression of this illness. Pharmacol. Res. 2018, 135, 1-11. [CrossRef] [PubMed]

28. Fedorova, L.V.; Tamirisa, A.; Kennedy, D.J.; Haller, S.T.; Budnyy, G.; Shapiro, J.I.; Malhotra, D. Mitochondrial impairment in the five-sixth nephrectomy model of chronic renal failure: Proteomic approach. BMC Nephrol. 2013, 14, 209. [CrossRef] [PubMed]

29. Yusof, H.M.; Cawood, A.L.; Ding, R.; Williams, J.A.; Napper, F.L.; Shearman, C.P.; Grimble, R.F.; Payne, S.P.; Calder, P.C. Limited impact of $2 \mathrm{~g}$ /day omega-3 fatty acid ethyl esters (Omacor(R)) on plasma lipids and inflammatory markers in patients awaiting carotid endarterectomy. Mar. Drugs 2013, 11, 3569-3581. [CrossRef]

30. Innes, J.K.; Calder, P.C. Marine Omega-3 (N-3) Fatty Acids for Cardiovascular Health: An Update for 2020. Int. J. Mol. Sci. 2020, 21, 1362. [CrossRef]

31. Chimienti, G.; Picca, A.; Sirago, G.; Fracasso, F.; Calvani, R.; Bernabei, R.; Russo, F.; Carter, C.S.; Leeuwenburgh, C.; Pesce, V.; et al. Increased TFAM binding to mtDNA damage hot spots is associated with mtDNA loss in aged rat heart. Free Radic. Biol. Med. 2018, 124, 447-453. [CrossRef] [PubMed] 